\title{
$H K$-band imaging polarimetry and radiative transfer modeling of the massive young stellar object CRL 2136
}

\author{
K. Murakawa, T. Preibisch, S. Kraus, and G. Weigelt
}

\author{
Max-Planck-Institut für Radioastronomie, Auf dem Hügel 69, 53121 Bonn, Germany \\ e-mail: murakawa@mpifr-bonn.mpg.de
}

Received 3 April 2008 / Accepted 6 August 2007

ABSTRACT

\begin{abstract}
Aims. We investigate the physical properties of the dust environment of the massive proto-stellar object CRL 2136 by means of two-dimensional radiative transfer modeling, which combines fitting of the spectral energy distribution, the intensity images, and the polarization images.

Methods. We obtained polarimetric images of CRL 2136 in the $H$ and $K$ bands using the CIAO instrument on the $8 \mathrm{~m}$ Subaru telescope. We developed a new Monte Carlo code which can deal with multiple-grain models and computes the SED, the dust temperature, and the Stokes IQUV images. With this code, we performed two-dimensional modeling of CRL 2136's circumstellar disk and envelope.

Results. Our images show a compact infrared source, two bright lobes extending towards the south and east, and two faint lobes extending towards the northwest and west. The polarization images show a polarization disk near the central star with a position angle of $\sim-135^{\circ}$, a polarization vector alignment approximately parallel to the polarization disk, and a region with low polarization between the eastern and the southern lobes. In our modeling, we assume three grain models: bare grains, warm grains with a crystalline water ice mantle, and cold grains with an amorphous water ice mantle. We obtained a maximum grain core size of $0.45 \mu \mathrm{m}$. We found that the CRL 2136 disk has a low disk mass of $0.007 M_{\odot}$, a large radius of $2000 \mathrm{AU}$, a scale height of 1.0, and a low accretion rate of $2.1 \times 10^{-7} M_{\odot} \mathrm{yr}^{-1}$ compared to an envelope mass infall rate of $1.0 \times 10^{-4} M_{\odot} \mathrm{yr}^{-1}$.

Conclusions. The predicted environment of the disk and the envelope is consistent with a scenario in which the central star forms rapidly $\left(\sim 2 \times 10^{5} \mathrm{yr}\right)$, with a high mass infalling rate, and nearly isotropically (large disk scale height) in the early phase. Then, the accretion of the disk matter is prevented by the strong radiation pressure from the luminous central star, resulting in a low disk mass and a low accretion rate.
\end{abstract}

Key words. stars: circumstellar matter - radiative transfer - polarization - stars: imaging

\section{Introduction}

CRL 2136 (=IRAS 18196-1331 = OH 17.6+0.2) is a massive star-forming region consisting of the near-infrared (NIR) source IRS 1 and a bipolar reflection nebula located at a distance of $2 \mathrm{kpc}$ (Kastner et al. 1992). IRS 1 possesses a protostar with a luminosity of $\sim 5 \times 10^{4} L_{\odot}$, and its mass was estimated to be higher than $10 M_{\odot}$ (Kastner et al. 1992). Previous NIR imaging polarimetry (e.g. Minchin et al. 1991; Kastner et al. 1992) detected two bright lobes of nebulosity extending east and south with respect to IRS 1 . Their polarization data shows a centrosymmetrically aligned pattern in the nebulosities surrounding IRS 1 and a linear alignment near IRS 1 with a position angle (PA) of $\sim 45^{\circ}$. Low resolution spectroscopy has detected deep absorption features of silicate at $9.7 \mu \mathrm{m}(\tau=5.07)$ and water ice at $3.07 \mu \mathrm{m}(\tau=2.72)$ (Willner et al. 1982). A CO emission line map shows blue- and red-shifted outflow components extending toward the southeast $\left(\sim 135^{\circ}\right)$ and the northwest $\left(\sim-45^{\circ}\right)$, respectively, approximately perpendicular to the direction of aligned polarization vectors near the central star (Kastner et al. 1994). The above-mentioned observational results are strong evidence for an optically thick torus, possibly a disk, and an envelope structure.

In recent years, some observational insights into the circumstellar environment of massive protostars have been obtained by high-resolution NIR imaging (e.g. Preibisch et al. 2003; Alvarez et al. 2004; Weigelt et al. 2006; Kraus et al. 2006) and radio interferometry (e.g. Menten \& van der Tak 2004; Patel et al. 2005; Steinacker et al. 2006). Furthermore, the circumstellar environment of young stellar objects (YSOs) is also known to be a molecule-forming region. Various absorption features, such as water ice, formaldehyde, and methanol, have been detected in the circumstellar environment (e.g. van Dishoeck 2004; Gibb et al. 2004). These molecules are thought to form on dust grains, which work as a catalyst to promote chemical reactions (e.g. Jones \& Williams 1984). The chemical reactions (or molecule formation) depend on physical conditions such as the dust temperature and the dust and gas densities. Therefore, investigating the physical properties of the dusty environment around young stellar objects is important in order to obtain a better understanding of the formation of massive stars as well as the chemical evolution in their surroundings.

Imaging polarimetry in the optical to NIR regime is a powerful technique to probe the dust environment because scattered polarized light may contain important information on the dust properties. Previous radiative transfer modeling successfully explained a polarization disk around the central star and a centrosymmetric polarization vector pattern in the envelope, which are often detected in the observed data of YSOs, applying disk and envelope geometries (e.g. Bastien \& Ménard 1988; Kenyon et al. 1993b; Whitney \& Hartman 1993; Fischer et al. 1996; Whitney et al. 1997; Lucas \& Roche 1998). Furthermore, polarization analysis allows us to constrain the grain size, particularly in the nebula on a large scale better than using the SED and the 
intensity images (e.g. Dougados et al. 1990; Pendleton et al. 1990; Murakawa et al. 2007, 2008a). In this work, we obtain NIR polarimetric images of the massive protostar CRL 2136 using the $8 \mathrm{~m}$ Subaru telescope and apply the above method. Although the above previous polarization analyses have mainly reproduced the intensity and polarization images, we attempt to fit the SED in our experiment as well. This allows us to better constrain the disk structure and grain size and to analyze the dust temperature distribution, particularly in the disk. We have already performed a similar technique for the proto-planetary nebula Frosty Leo (Murakawa et al. 2008a). In Sect. 2, we present our observational results. For our radiative transfer modeling, we developed a Monte Carlo code which can analyze polarized light and handle multiple-grain models that are distributed arbitrarily within the model geometry. We summarize the algorithms of our code in Sect. 3. In Sect. 4, the details of our modeling and the results are presented.

\section{HK-band imaging polarimetry}

\subsection{Observations}

We obtained $H$ - and $K$-band polarimetric images using the NIR camera CIAO on the $8 \mathrm{~m}$ Subaru telescope on 2005 June 28. The central wavelengths and the band widths of the $H$ - and $K$-band filters are $\lambda_{H}=1.65 \mu \mathrm{m}$ and $\Delta \lambda_{H}=0.30 \mu \mathrm{m}$, and $\lambda_{K}=2.20 \mu \mathrm{m}$ and $\Delta \lambda_{K}=0.34 \mu \mathrm{m}$, respectively. We used a medium resolution camera with a pixel scale of $0.0217 \operatorname{arcsec}$ pix $^{-1}$. The natural seeing was $\sim 00^{\prime} 4$ at $R$ band. Since the target is faint at the wavelength for wavefront sensing $\left(m_{R}>16 \mathrm{mag}\right)$ and there is no bright single star near the target, we did not apply the adaptive optics correction. In order to measure linear polarization, we obtained four sets of images with half-wave plate (HWP) orientations of $0^{\circ}, 45^{\circ}, 22.5^{\circ}$, and $67.5^{\circ}$. At each orientation of the HWP, two frames with $30 \mathrm{~s}$ exposure times and five frames with $3 \mathrm{~s}$ were obtained, and 8 and 12 jitter positions were applied in the $H$ and $K$ bands, respectively. The total integration times were $32 \mathrm{~min}$ for the $H$ band and $12 \mathrm{~min}$. for the $K$ band. Although we also obtained intensity images in the $J$ band, we do not discuss the results due to their poor image quality. For flux and polarization calibrations, we observed Elias 2-14 and BS 6141. We found an offset of $2^{\circ}$ in position angle of the polarization orientation and a difference of $0.3 \%$ in the degree of polarization, which are both within the measurement errors.

We reduced the observed data following the procedure described in our previous papers (Murakawa et al. 2004, 2005). After subtraction of the dark frame and flat-fielding, we obtained the Stokes $I Q U$ images, the degree of linear polarization $(P)$, the polarization position angle $(\theta)$, as well as their error images. We found signal-to-noise ratios of the Stokes $I$ (intensity) images of 30-120 per pixel and an error of linear polarization of $<15 \%$ per pixel in the region with surface brightnesses higher than $\sim 2 \times 10^{-16}$ and $\sim 1.5 \times 10^{-15} \mathrm{Wm}^{-2} \mu \mathrm{m}^{-1} \operatorname{arcsec}^{-2}$ in the $H$ and $K$ bands, respectively. The uncertainty on the polarization position angle per pixel is $<10^{\circ}$ in these regions. The average PSF size is measured to be 0.35 in the $H$ and $K$ bands from several point-source-like features detected in our images.

\subsection{Results}

Figure 1 presents the intensity images overlaid with polarization vector lines and the degree of polarization images in the $H$ (left) and $K$ (right) bands. The field of view (FOV) is $19^{\prime \prime} \times 19^{\prime \prime}$. Figure 2 shows a sketch of CRL 2136, which illustrates some components detected in our data and a $\mathrm{CO}$ emission line observation and inferred from our modeling. Our images show the bright infrared source IRS 1, fan-shaped nebulosities extending toward the south, east, west, and northwest, and several point sources. The polarimetric data shows a centro-symmetric vector pattern in the nebulosities. The degree of polarization reaches values up to $\sim 30 \%$ in the $H$ band and $\sim 50 \%$ in the $K$ bands. Near IRS 1, we see a polarization disk extending at a position angle (PA) of $\sim-135^{\circ}$, but it is not clear in the northeast. In this region, polarization vector lines are systematically aligned along a PA of $\sim 30^{\circ}$. However, in the $K$ band data, they converge at a point, i.e. the polarization null point, in the southwest. These features are consistent with previous results and they led authors to support the disk hypothesis (Minchin et al. 1991; Kastner et al. 1992).

Our images clearly resolve the southern component into two lobes; i.e. the west $\mathrm{S} 1$ and the east $\mathrm{S} 2$ lobes. While high polarizations are detected in the $\mathrm{S} 1$ and $\mathrm{E}$ lobes $\left(P_{K} \sim 50 \%\right)$, the $\mathrm{S} 2$ lobe as well as a fan-shaped region between the E and $\mathrm{S} 1$ lobe have low polarizations of $P_{H} \lesssim 15 \%$ and $P_{K} \lesssim 30 \%$. If we assume an envelope with a polar cavity structure, the E and S1 lobes and the fan-shaped region correspond to the projected edges and inner part of the cavity, respectively. Furthermore, we measured the polarizations to be $P_{H}=32 \%$ and $P_{K}=21 \%$ at IRS 1 using a $0^{\prime} .7$ diameter aperture, and $P_{H} \lesssim 15 \%$ and $P_{K} \lesssim 10 \%$ southwest of IRS 1 in the polarization disk. We stress that the $H$-band polarization in these regions is higher than in the $K$ band, in contrast to the envelope. These results are in rough agreement with previous $3 \mu \mathrm{m}$ spectropolarimetry (Hough et al. 1989; Holloway et al. 2002). Details of our interpretation of the above observational results based on our radiative transfer modeling will be discussed in Sect. 4.2.2.

\section{Radiative transfer code}

We developed a new Monte Carlo radiative transfer code stsh (StrahlungsTransportcode für Staubige Hüllen) and used it to investigate the physical properties of the CRL 2136 nebula. Our code is designed to simulate scattering, absorption, and reemission of radiation in the model dust shell under local thermal equilibrium. It can treat transfer of polarized light and multiple-grain models in a model geometry. The code computes the SED and the dust temperature for multiple-grain models and generates the Stokes $I Q U V$ images. The principles of radiative transfer and the Monte Carlo method have already been discussed in several papers (e.g. Cashwell \& Everett 1959; Witt 1977; Yusef-Zadeh et al. 1984; Ivezić \& Elitzur 1997; Lucy 1999; Bjorkman \& Wood 2001; Niccolini et al. 2003). In this section, we summarize the algorithms of our code.

The model geometry consists of cells in one-dimensional (1-D) spherically symmetric coordinates, two-dimensional (2-D) axi-symmetric coordinates, or three-dimensional (3-D) spherical coordinates. For each cell, the dust density can be specified for each grain model. Our code currently treats spherical grains with an MRN- (Mathis et al. 1977) or KMH-like (Kim et al. 1994) size distribution with or without a single-layered mantle. Cross sections of absorption, scattering, and extinction, as well as scattering amplitude matrix elements are calculated using Mie's scattering theory. For bare grains, we use the algorithm reported by Wolf \& Voshchinnikov (2004), which can handle grains with size parameter ${ }^{1}$ values up to $\sim 10^{7}$. For coated grains,

\footnotetext{
1 The size parameter $x$ is defined to be $x=2 \pi a / \lambda$, where $a$ and $\lambda$ are the grain radius and the wavelength, respectively.
} 

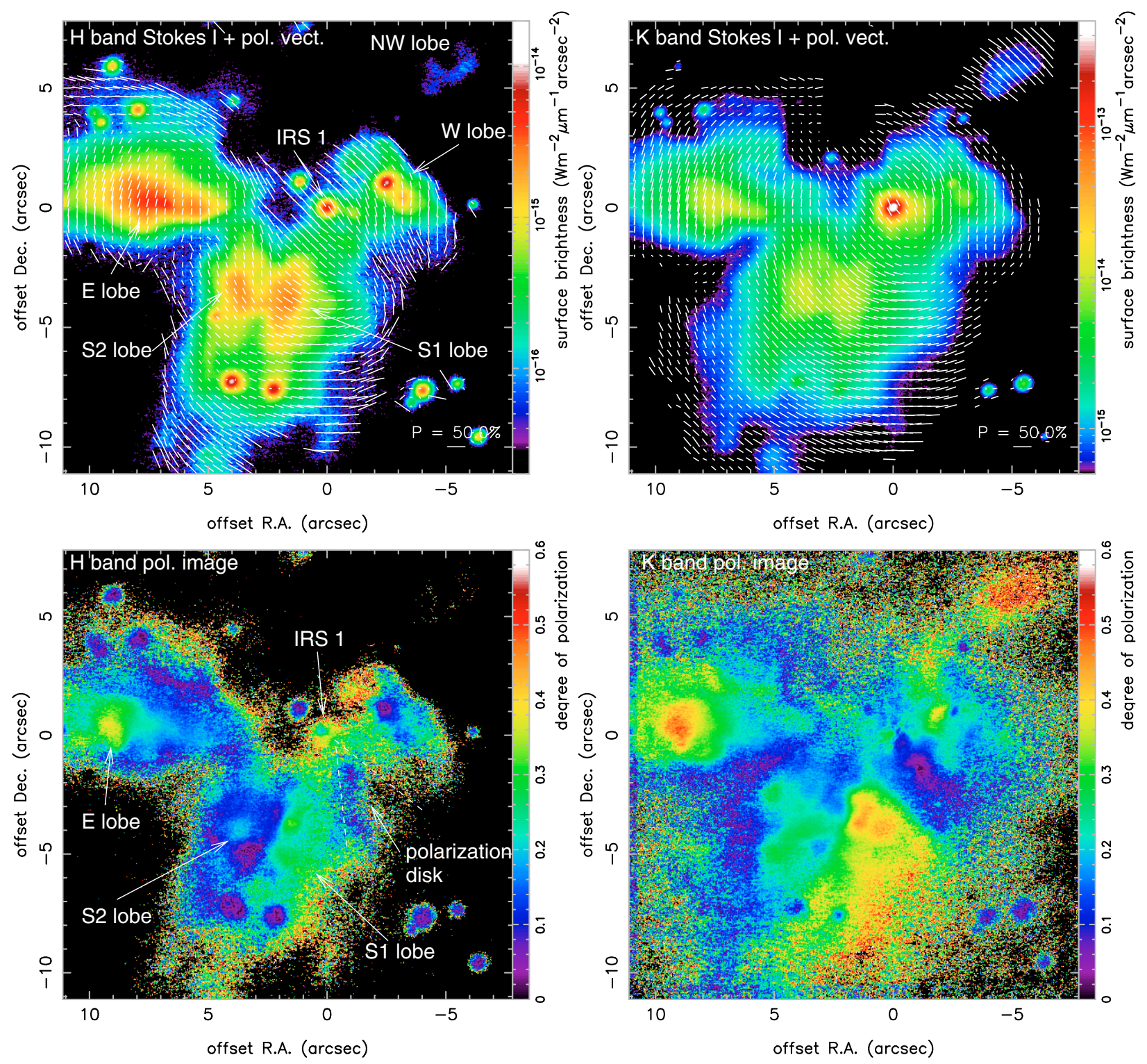

Fig. 1. Polarimetric images of CRL 2136. The top panels are the obtained intensity (Stokes $I$ ) images in the $H$ (left) and $K$ (right) bands with the polarization vector lines. The field of view is $19^{\prime \prime} \times 19^{\prime \prime}$. The bottom panels show the degree of linear polarization images in the $H(l e f t)$ and $K$ (right) bands. The position of the central star is indicated with the offset of $\left(00^{\prime} 0,0{ }^{\prime} 0\right)$.

we use the algorithm by Wiscombe (1980) and Nussenzveig \& Wiscombe (1980), which can treat a coating material even with high absorption. We combined this algorithm with Wolf \& Voshcninnikov's algorithm in order to treat a very large size parameter value. In each grain model, the cross sections and scattering amplitude matrix elements are averaged in size with a size distribution function $n(a)$ and in chemical composition with a specified fraction.

The radiation from the radiation source is split into several photon packets. The spectrum of the radiation source is given by a black-body spectrum with an effective temperature $T_{\text {eff }}$ or by an arbitrary SED specified with an external file. The former case is used for a single stellar system. When a photon packet is emitted, the wavelength is determined following Eqs. (14)-(16) in the paper by Bjorkman \& Wood (2001). The latter case is used for a non-black-body radiation source, such as close multiple stellar systems and active galactic nuclei (AGNs). The radiation from the source is assumed to be unpolarized and to be emitted isotropically.
A photon packet propagates straight ahead in the model geometry to the next interacting point, which is chosen by $\tau=$ $-\ln z$ with a uniformly distributed random number $z \in(0,1]$. At an interacting point, a grain model to interact is determined. The probability of choosing the $j$ th grain model is given by $\rho_{i, j}\left(\kappa_{j}+\sigma_{j}\right) / \sum_{j} \rho_{i, j}\left(\kappa_{j}+\sigma_{j}\right)$, where the quantities of $\rho, \kappa$, and $\sigma$ are the dust number density and the cross sections of absorption and scattering, respectively. Then, with the albedo $\omega_{j}=\sigma_{j} /\left(\kappa_{j}+\sigma_{j}\right)$ of the selected grain model, absorption $\left(z>\omega_{j}\right)$ or scattering $\left(z \leq \omega_{j}\right)$ is determined with a random number $z \in[0,1)$. When the scattering event is chosen, the direction of the photon propagation is changed. Our code uses Fischer's algorithm (Fischer et al. 1996), which determines the direction of scattered light depending on the polarization status before scattering. The Stokes parameters after scattering are updated with the scattering Mueller matrix (Bohren \& Huffmann 1983). When the absorption event is chosen, the dust temperature is updated and a new wavelength of reemitted light is determined. Our 


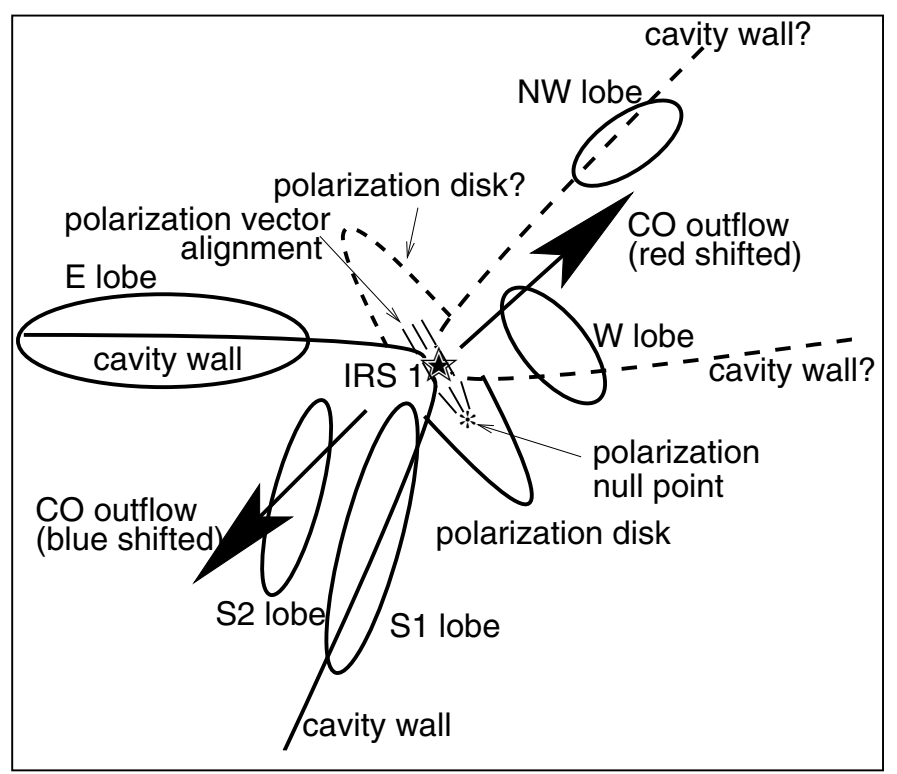

Fig. 2. Cartoon of CRL 2136 illustrating the features detected in our data, the direction of the $\mathrm{CO}$ outflow, as well as our interpretation of a disk and envelope structure. The polarization disk on the northeastern side of IRS 1 is not visible in our data.

code employs Bjorkman \& Wood's method (Bjorkman \& Wood 2001). After the interaction, the photon packet performs further random walks until it leaves the model geometry or the number of times of interaction reaches the specified limit. If the photon packet reaches the outer boundary of the model geometry and the direction of the ray points towards the observer, the SED and/or the Stokes images are/is updated with the photon information. Then, a new photon packet is emitted from the radiation source. The above procedures are repeated until all photon packets are examined.

It is well known that the images generated by the above algorithm (hereafter the standard full interacting mode) suffer from severe Monte Carlo noise in most actual experiments. In order to relieve this problem, the concept of weighted photons and its applications of the forced first scattering (Cashwell \& Everett 1959; Witt 1977) and peeling off (Yusef-Zadeh et al. 1984; Wood \& Reynolds 1999; Gordon et al. 2001) have been proposed. Dramatic improvements of the quality of the model images are expected in cases of models with a high extinction (e.g. $\tau>100)$ and a large scale height value (e.g. $H>0.5$ ) or with a 3-D geometry. Our code uses these algorithms.

We have tested our code by comparing several model results with those obtained using the DUSTY code (Ivezić \& Elitzur 1997) for the 1-D case and the mcsim_mpi code (Ohnaka et al. 2006) for the 2-D case. The 3-D case was tested by choosing an axi-symmetric model geometry which can be compared with the results obtained in a 2-D simulation. In all cases, we found that the results of SED, dust temperature, and Stokes IQUV images produced by our code are in good agreement with those obtained using the above codes.

\section{Modeling the CRL 2136 nebula}

\subsection{Model assumptions}

\subsubsection{Stellar parameters}

The distance to CRL 2136 is not well known. Allen et al. (1977) suggested $5 \mathrm{kpc}$, although this result was not well justified. We assume a kinematic distance of $D=2 \mathrm{kpc}$ (Kastner et al. 1992). For this distance, Kastner et al. (1992) estimated the stellar luminosity to be $\sim 5.5 \times 10^{4} L_{\odot}$ based on the MIR flux, which dominates the SED. We set the luminosity to be this value. The stellar temperature is also not well known. Harvey et al. (2000) adopted $T_{\star}=33000 \mathrm{~K}$ from theoretical predictions for a zeroage main-sequence star of the above luminosity (e.g. Bernasconi \& Maeder 1996; Yorke \& Sonnhalter 2002). However, the star could be cooler than the above temperature because of the lack of radio continuum emission (e.g. Harvey et al. 2000) and weak or non-detection of the $\mathrm{H}_{2}$ 1-0 S(1) emission line towards this object (Kastner et al. 1992; Kastner \& Weintraub 1996). They estimated the stellar temperature to be $7000-15000 \mathrm{~K}$. We set $T_{\star}$ to be $20000 \mathrm{~K}$, which is an intermediate value of the above estimates. An uncertainty of $\sim 10$ thousand Kelvins is possible. Even such a large uncertainty does not affect the model results much because the wavelength ranges in the NIR or longer are in the Rayleigh-Jeans law regime. We adopt a stellar mass of $M_{\star}=20 M_{\odot}$, which is expected from the above-mentioned theoretical predictions.

\subsubsection{Dust grains}

Dust in star formation regions is often modeled similarly to the interstellar matter (ISM); i.e. two major chemical compositions of silicate and graphite and a power law size distribution (Mathis et al. 1977; Draine \& Lee 1984; Kim et al. 1994). However, variations of grain properties from region to region and even within one region have been reported (e.g. Cardelli et al. 1989; Clayton et al. 2003; Larson \& Whittet 2005). Since the dust in CRL 2136 has not yet been investigated in detail, we assume a DL-MRN model; i.e. a mixture of grain cores of astronomical silicate and of graphite with fractions of $62.5 \%$ and $37.5 \%$, respectively (Draine \& Lee 1984). Although some evidence for the presence of non-spherical grains has been reported (Hough et al. 1989; Holloway et al. 2002), we assume spherical grains for simplicity. We assume a size distribution with a power index given by $n(a) \propto a^{-3.5}$. The minimum grain size $a_{\text {min }}$ does not affect the SED and the polarization properties much, whereas the maximum size $a_{\max }$ can have a strong influence. Therefore, we set a fixed value of $a_{\mathrm{min}}=0.005 \mu \mathrm{m}$, (corresponding to the diffuse ISM population, Mathis et al. 1977) and estimate $a_{\max }$ from our modeling.

Numerous molecules such as $\mathrm{H}_{2} \mathrm{O}$ (Willner et al. 1982), $\mathrm{CH}_{3} \mathrm{OH}$ (Skinner et al. 1992), cyano group (often referred to as "XCN", Pendleton et al. 1999; Whittet et al. 2001), and CO (Skinner et al. 1992) have been detected towards CRL 2136. These volatile molecules are thought to form on the grain surface and cover the refractory grain core as a mixture or "dirty icy" mantles (e.g. Tielens \& Hagen 1982; Jones \& Williams 1984; Herbst \& Leung 1985). Water ice is the major component and is an important tracer that allows us to derive information about the dust temperature and grain size from spectroscopic data. To reduce complexity, we consider only water ice as grain mantles. It is known that water ice stays in a crystalline form at temperatures between $\sim 80 \mathrm{~K}$ and the sublimation temperature of $\sim 140 \mathrm{~K}$ or in an amorphous form below $\sim 80 \mathrm{~K}$ (Hagen et al. 1981; Leger et al. 1983). Therefore, we take into account three grain species: grains with an amorphous ice mantle, grains with a crystalline ice mantle, and bare grains at temperatures between $\sim 140 \mathrm{~K}$ and the dust sublimation temperature of $\sim 1500 \mathrm{~K}$. Details about our grain modeling are described in Sect. 4.2.1. 


\subsubsection{Disk and envelope geometries}

As discussed in Sect. 2, several observational results of CRL 2136 can be explained qualitatively with a disk and envelope structure. Therefore, we applied both of these geometries in our radiative transfer modeling using a dust density distribution given,

$\rho=\rho_{\text {disk }} f_{1}+\rho_{\text {env }} f_{2}$,

where $f_{1}$ and $f_{2}$ are Fermi-type filter functions to guarantee a smooth transition between the disk and the envelope components (Fischer et al. 1996).

For the disk structure, we examine a Keplerian rotating disk in hydrodynamical equilibrium (labeled "SH", Shakura \& Sunyaev 1973; Pringle 1981). Using the 2-D cylindrical coordinate $(r, z)$, the density structure is given by

$$
\begin{aligned}
\rho_{\text {disk }}^{\mathrm{SH}}(r, z) & =\rho_{\mathrm{d}}\left(r / R_{\mathrm{disk}}\right)^{-\alpha} \exp \left(-\frac{\pi}{4}\left[\frac{z}{h(r)}\right]^{2}\right), \\
h(r) & =R_{\text {disk }} H\left(r / R_{\text {disk }}\right)^{\beta},
\end{aligned}
$$

where $\rho_{\mathrm{d}}$ and $H$ are the mass density at the disk radius $R_{\text {disk }}$ in the equatorial plane and the scale height of the disk, respectively. The mass density coefficient $\rho_{\mathrm{d}}$ is determined with the disk mass $M_{\text {disk }}$. The values of $\alpha$ and $\beta$ are $15 / 8$ and $9 / 8$, respectively. While the above model has a flared shape because of $\beta>1$, Lazareff et al. (1990) proposed another disk model (massive disk, "MD") which is a potential model for proto-stellar objects. The density distribution is given by

$\rho_{\text {disk }}^{\mathrm{MD}}(r, z)=\rho_{\mathrm{d}}\left(r / R_{\text {disk }}\right)^{-\alpha} \exp \left(-\left|\frac{z}{h(r)}\right|\right)$.

This model is classified into four sub classes, depending on the values of $\alpha$ and $\beta$. These are characterized by azimuthal velocity and the surface density $\Sigma$ and have $(\alpha, \beta)$ values of $(2,1)$, $(1,1),(0,1)$, and $(0,0)$ for the Toomre model (TO), the Larson model (LA), the rigid body model (RB), and the infinite isothermal slab model (SL), respectively (Toomre 1982; Larson et al. 1984; Fischer et al. 1996).

In our model, the inner radius $R_{\mathrm{in}}$ is set to be the dust sublimation radius of $30 \mathrm{AU}$, which is appropriate for a star with an estimated luminosity of $5.5 \times 10^{4} L_{\odot}($ e.g. Monnier \& Millan-Gabet 2002). We determine the three other free parameters of the disk radius $R_{\text {disk }}$, the disk mass $M_{\text {disk }}$, and the disk scale height $H$ from our modeling.

For the envelope, we use the model of the slowly rotating, infalling envelope (Ulrich 1976; Cassen \& Moosman 1981; Terebey et al. 1984). The mass density distribution is given by

$\rho_{\mathrm{env}}(R, \mu)=\frac{\dot{M}_{\mathrm{env}}}{4 \pi \sqrt{G M_{\star} R^{3}}}\left(1+\frac{\mu}{\mu_{0}}\right)^{-1 / 2}\left(\frac{\mu}{\mu_{0}}+\frac{2 \mu_{0}^{2} R_{\mathrm{c}}}{R}\right)^{-1}$,

where $R, G, \dot{M}_{\text {env }}$, and $R_{\mathrm{c}}$ are the radial distance from the central star, the gravitational constant, the envelope mass infall rate, and the centrifugal radius, respectively. Beyond $R_{\mathrm{c}}$, the dust density of this envelope is approximately proportional to $R^{-1.5}$. An FIR observation and modeling satisfies this result (Harvey et al. 2000). The quantity $\mu$ is the cosine of the polar angle $\mu=\cos \theta$, and $\mu_{0}$ is the angle of a stream line as related through the equation

$\mu_{0}^{3}+\mu_{0}\left(R / R_{\mathrm{c}}-1\right)-\mu\left(R / R_{\mathrm{c}}\right)=0$.
The polar cavity is the region for $\mu>\mu_{0}$. The density inside the cavity is reduced to 0.001 compared to the surrounding envelope.

If we assume for CRL 2136 that the E and S1 lobes represent the cavity edge, the opening angle is $65^{\circ}$, i.e. $\mu_{0}=\cos \left(65^{\circ} / 2\right)$. The centrifugal radius $R_{\mathrm{c}}$ is set to be $R_{\text {disk. }}$. The outer radius $R_{\text {out }}$ of the envelope affects mainly the fluxes in the FIR and longer wavelengths and was estimated to be $0.3 \mathrm{pc}$, which is about the extension of the submillimeter continuum at $800 \mu \mathrm{m}$ (Kastner et al. 1994). Therefore, we began to use $0.3 \mathrm{pc}$ as the initial value and changed it later in the course of the modeling process. A free parameter in our modeling is the envelope mass infall rate $\dot{M}_{\text {env }}$. The value is searched around an order of $10^{-4} M_{\odot} \mathrm{yr}^{-1}$, which is the typical value for massive YSOs (Wolfire \& Cassinelli 1986).

\subsection{Numerical simulations}

Although some of the dust and geometry parameters were estimated in earlier studies, a large number of parameters still have not been determined. Because it is unrealistic to scan the whole parameter space, we follow the following procedure. First, we constrain the dust grain parameters by fitting the polarization maps and the spectrum. Then, with the best grain model, we compute SEDs in order to obtain some potential solutions. We produce the intensity and polarization images of these models and, finally, choose a good model.

\subsubsection{Grain model}

As described in Sect. 4.1.2, we take into account three grain species: cold grains (with an amorphous water ice mantle), warm grains (with a crystalline water ice mantle), and bare grains. We first examine the cold grains, which probably exist in the lowtemperature envelope at large distances from the central star. While the color information in our $H$ - and $K$-band intensity images depends on the grain size and the dust density (or the optical depth), the degree of polarization is mostly governed by only the grain size since the scattering angle dependence vanishes towards the projected cavity, i.e. the E and S1 lobes, by integrating along the line of sight. Furthermore, the degree of polarization varies the most as a function of the size parameter $x$ around 1 , which corresponds to submicron-sized grains at NIR wavelengths. Such grain sizes are very likely in the envelope. Therefore, we determine the grain size $a_{\max }$ by polarization fit. We compare the polarization data in the above regions, where the dust density distribution in the plane which crosses the central star the scattering matter (i.e. dust), and the observer, can be approximated with a 1-D spherically symmetric geometry. In addition, the polarization vectors are aligned centro-symmetrically in the above $\mathrm{E}$ and $\mathrm{S} 1$ lobes. Thus, we apply a single scattering analysis (Dougados et al. 1990). In this modeling, light emitted from the central star is scattered only once in a 1-D dust shell and contributes to the observed 1-D image. The degree of polarization is calculated, using the Stokes $I Q$ parameters, by $-Q / I$. Although the grains in the nebula are expected to be covered with a water ice mantle, the mantle does not affect the scattering properties much if the mantle is not too thick compared to the grain core radius or the considered wavelength is not at the absorption bands, e.g. $3.1 \mu \mathrm{m}$. Therefore, we modeled bare grains and examined values for $a_{\max }$ between 0.2 and $1.0 \mu \mathrm{m}$ in increments of $0.1 \mu \mathrm{m}$. For the geometry parameters, we set the inner radius $R_{\text {in }}$, the outer radius $R_{\text {out }}$, and the power index of the dust density distribution to be $30 \mathrm{AU}, 0.3 \mathrm{pc}$, and -1.5 , respectively. Because the polarization at a large separation from the central 


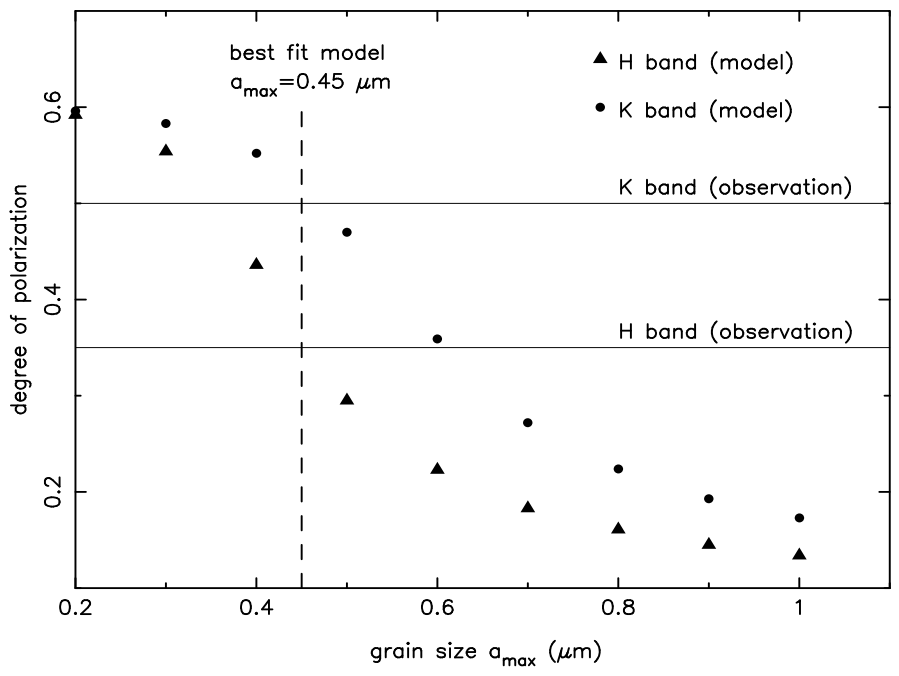

Fig. 3. The degree of polarization dependence on the grain size $\left(a_{\max }\right)$. The polarization is calculated by means of a single scattering modeling with a spherically symmetric dust shell. For simplicity, bare grains are assumed in this modeling. The minimum grain size $a_{\min }$ and the power index of the size distribution are fixed to be $0.005 \mu \mathrm{m}$ and -3.5 , respectively.

star varies little in optically thin cases, we set the optical depth at $V$ band to be 1. Figure 3 shows the degree of polarization $5^{\prime \prime}$ from the central star as a function of grain size $a_{\max }$. We find that a grain model with $a_{\max }=0.45 \mu \mathrm{m}$ reproduces our observational results of $P_{H} \sim 30 \%$ and $P_{K} \sim 50 \%$ quite well.

We determine the thickness of water ice mantles of the cold and warm dust and the grain core size of the warm dust by fitting the $3 \mu \mathrm{m}$ spectrum. It is well known that the $3 \mu \mathrm{m}$ water ice absorption feature suggests the dust temperature from the width of the absorption feature, the chemical composition from the $3.4 \mu \mathrm{m}$ feature, and the dust grain size from the bottom position in the wavelength (e.g. Smith et al. 1989). In principle, it is possible to investigate these parameters with the $3 \mu \mathrm{m}$ spectroscopic data. However, we simplified our modeling to treat only water ice. Thus, our modeling fits only the shape of the spectrum near $3.08 \mu \mathrm{m}$ and estimates the fractions of cold and warm water ice mantles and the grain sizes. Although bare grains are expected to exist in the line of sight towards the central star, their optical properties affect the water ice absorption feature only marginally. Thus, we model a mixture of the cold and warm dust. The mantle thickness of water ice is obtained with the mantle/core volume ratio of $f_{\mathrm{c}}$ for the cold grains and $f_{\mathrm{w}}$ for the warm grains, respectively. We use optical constants provided by Bertie et al. (1969) for crystalline and Leger et al. (1983) for amorphous water ice. We calculate the extinction spectra as a function of wavelength of the model grains and compare them with observations reported by Holloway et al. (2002). The best grain model was found to be $f_{\mathrm{c}}=0.05, a_{\min }=0.005 \mu \mathrm{m}$, $a_{\max }=0.4 \mu \mathrm{m}, f_{\mathrm{w}}=3.0$, and the fraction of the cold grains $w=0.1$. Figure 4 compares the observed $3 \mu \mathrm{m}$ water ice absorption feature with our model result. The model profile at $\lambda<3.0 \mu \mathrm{m}$ and $\lambda>3.2 \mu \mathrm{m}$ is wider than the observation. This discrepancy is mainly due to contamination by other molecules in the real grain mantles such as the $\mathrm{C}-\mathrm{H}$ group, i.e. "dirty ice" (Merrill et al. 1976; Duley \& Williams 1981; d'Hendecourt \& Allamandola 1986; Preibisch et al. 1993). A range of $a_{\max }$ up to 0.8 and $f_{\mathrm{w}}$ down to 1.0 provides acceptable fits. This estimation is in rough agreement with the one provided by Dartois et al. (2002). Since the estimated size of warm grains does not

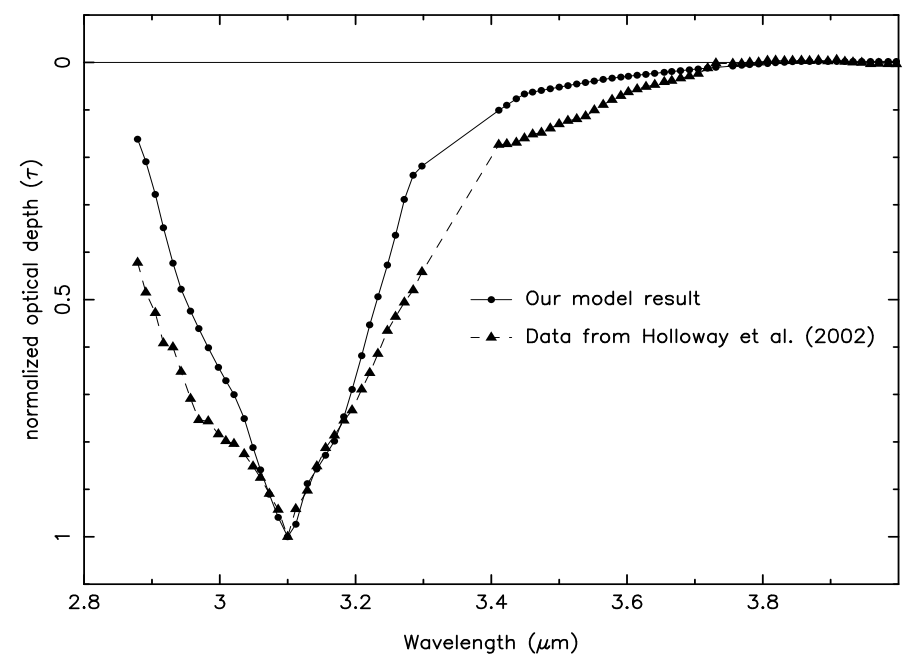

Fig. 4. Comparison of the normalized optical depths of $3 \mu \mathrm{m}$ water ice absorption feature of the model result (filled triangles with dashed line) with an observational result (filled circles with solid line). The observational data was scanned from the paper by Holloway et al. (2002).

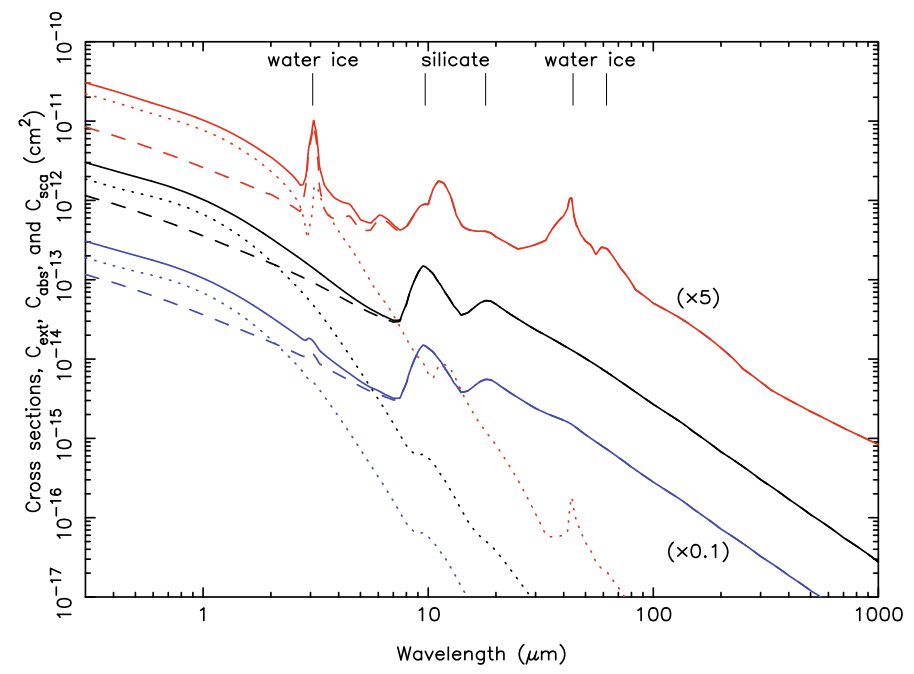

Fig. 5. Cross sections of extinction (solid line), scattering (dashed line), and absorption (dotted line) of the modeled grains as a function of wavelength. Black, red, and blue lines denote the bare grains $(0.005 \leq a \leq 0.45 \mu \mathrm{m})$, warm grains with a crystalline ice mantle $\left(0.005 \leq a \leq 0.45 \mu \mathrm{m}, f_{\mathrm{w}}=300 \%\right)$ (multiplied by a factor of 5 ), and cold grains with an amorphous ice mantle $(0.005 \leq a \leq 0.45 \mu \mathrm{m}$, $f_{\mathrm{c}}=5 \%$ ) (multiplied by a factor of 0.1 ), respectively.

sufficiently differ from the grain size of the cold grains, we adopt $0.45 \mu \mathrm{m}$ for our further modeling. We also assume the same core size for bare grains. This will be revisited later if necessary. Figure 5 shows cross sections of the modeled grains. Black, red, and blue lines denote the bare grains, warm grains, and cold grains, respectively. Scattering cross sections dominate up to $3 \mu \mathrm{m}$. In each profile, silicate features at $10 \mu \mathrm{m}$ and $18 \mu \mathrm{m}$ are seen. Warm grains with a crystalline ice mantle exhibit the characteristic feature at $62 \mu \mathrm{m}$, which does not appear in the amorphous form. 


\subsubsection{Monte Carlo experiments}

Because the real CRL 2136 nebula exhibits highly complicated features, we do not expect our 2-D model to explain every aspect of the observed SED and intensity and polarimetric images. Instead, we attempt to reproduce features which characterize important structures of the modeled disk and envelope. Before scanning parameter spaces, we examined some parameter sets in order to obtain rough solutions and to investigate the effects of the parameters on the model results. From this study, we determined the following parameter ranges: a disk radius $R_{\text {disk }}$ between 1000 and $5000 \mathrm{AU}$ in increments of $1000 \mathrm{AU}$; a disk mass $M_{\text {disk }}$ of $0.007,0.01,0.02,0.03,0.05,0.07$, and $0.1 M_{\odot}$; a disk scale height $H$ of $0.2,0.5,1.0$, and 2.0; and an envelope mass infall rate $\dot{M}_{\text {env }}$ of $7.0 \times 10^{-5}, 1.0 \times 10^{-4}, 1.5 \times 10^{-4}$, and $2.0 \times 10^{-4} M_{\odot} \mathrm{yr}^{-1}$. The gas-to-dust mass ratio is assumed to be 100 . For the outer radius $R_{\text {out }}$, we examine $0.3,0.5$, and $1.0 \mathrm{pc}$ to find which value provides a better fit of the flux in the bipolar lobes in the $H$ - and $K$-band images and those in the submillimeter wavelength range. The viewing angles $\theta_{\mathrm{v}}$ were chosen to be $60^{\circ}, 70^{\circ}, 75^{\circ}, 80^{\circ}$, and $90^{\circ}$ (edge-on). The dust temperature in the inner radius is estimated to be about $1500 \mathrm{~K}$, which is consistent with the dust sublimation temperature. The radii where the dust temperature reaches $140 \mathrm{~K}$ and $80 \mathrm{~K}$ were found to be $\sim 1500 \mathrm{AU}(\mathrm{SH}, \mathrm{TO}), \sim 1800 \mathrm{AU}$ (LA), and $\sim 5000 \mathrm{AU}$ for all disk models. Because these values vary only insignificantly in the above-examined parameter space, we fixed them in our subsequent simulations. For the above 1680 parameter sets for each disk model, we computed the SEDs to constrain the above parameters. This SED fitting was done by eye. From this survey, we found that a large disk radius $\left(R_{\text {disk }}>3000 \mathrm{AU}\right)$, a low disk mass $\left(M_{\text {disk }}<0.007 M_{\odot}\right)$, or a low disk scale height $(H<1.0)$ result in a too shallow $10 \mu \mathrm{m}$ silicate feature or a too high flux in the optical and NIR because of a too low optical depth. On the other hand, a small disk radius $\left(R_{\text {disk }}<2000 \mathrm{AU}\right)$ or a high disk mass $\left(M_{\text {disk }}>0.01 M_{\odot}\right)$ causes the opposite results. For the envelope parameters, the lower the infalling rate or smaller the outer radius is, the lower the FIR to submillimeter fluxes and the lower surface brightness in the nebulosity in the NIR become. The viewing angle affects the optical depth in the line of sight towards the central star and the appearances of the lobes and the polarization distribution, particularly in the projected inner part of the cavity. A viewing angle close to $90^{\circ}$ fits better in polarization, but the optical and NIR fluxes become too low if the viewing angle is larger than $75^{\circ}$. On the other hand, a viewing angle of $\theta_{\mathrm{v}}<70^{\circ}$ causes $10 \mu \mathrm{m}$ silicate features to be too shallow. Furthermore, the SH, TO, and LA disk models produce acceptable results, but the RB and SL fail, as discussed in Sect. 4.2.3. The reasonable parameters were found to be $R_{\text {disk }}=2000-3000$ AU, $M_{\text {disk }}=0.007-0.07 M_{\odot}, H=1.0$ $2.0, \dot{M}_{\text {env }}=1.0-1.5 \times 10^{-4} M_{\odot} \mathrm{yr}^{-1}$, and $R_{\text {out }}=0.3-0.5 \mathrm{pc}$, and $\theta_{\mathrm{v}}$ of $70^{\circ}-75^{\circ}$. We generated Stokes $I Q U V$ images in the $H$ and $K$ bands and, finally, selected a parameter set for each disk model which reproduces the characteristic features seen in the observed intensity and polarization images. The parameters of the selected model for each disk model are: $R_{\text {disk }}=2000 \mathrm{AU}$, $M_{\text {disk }}=0.007 M_{\odot}(\mathrm{SH}, \mathrm{TO})$ and $0.05 M_{\odot}(\mathrm{LA}), H=2.0(\mathrm{SH})$ and $H=1.0$ (TO, LA), $\dot{M}_{\text {env }}=1.0 \times 10^{-4} M_{\odot} \mathrm{yr}^{-1}, R_{\text {out }}=0.5 \mathrm{pc}$, and $\theta_{\mathrm{v}}=70^{\circ}$. In these disk models, we found that the TO model is favorable. We also estimated the disk accretion rate $\dot{M}_{\text {acc }}$ and the total envelope mass to be $2.1 \times 10^{-7} M_{\odot} \mathrm{yr}^{-1}$ using Eq. (5) in Whitney et al. (2003) and $177 M_{\odot}$, respectively, and the visual optical depth towards the central star at the chosen viewing angle to be 66 (SH), 77 (TO), and 78 (LA). The parameters, the
Table 1. Parameters of our 2-D radiative transfer model of CRL 2136.

\begin{tabular}{|c|c|c|}
\hline Parameters & Adopted values & Comments $^{1}$ \\
\hline \multicolumn{3}{|c|}{ Central star (IRS 1$)^{2}$} \\
\hline$T_{\star}$ & $20000 \mathrm{~K}$ & Adopted \\
\hline$\hat{L_{\star}}$ & $55000 L_{\odot}^{3}$ & Adopted \\
\hline$M_{\star}$ & $20 M_{\odot}^{4}$ & Adopted \\
\hline$d$ & $2 \mathrm{kpc}^{5}$ & Adopted \\
\hline$R_{\star}$ & $5 \times 10^{11} \mathrm{~cm}$ & Calculated \\
\hline \multicolumn{3}{|c|}{ Bare grains $^{6}$} \\
\hline Size & $0.005 \leq a \leq 0.45 \mu \mathrm{m}, n(a) \propto a^{-3.5}$ & \\
\hline Region & $140 \lesssim T_{\mathrm{d}} \lesssim 1500 \mathrm{~K}^{7}$ & \\
\hline \multicolumn{3}{|c|}{ Warm grains $^{6}$} \\
\hline Size & \multirow{3}{*}{\multicolumn{2}{|c|}{$\begin{array}{l}0.005 \leq a \leq 0.45 \mu \mathrm{m}, n(a) \propto a^{-3.5} \\
\text { crystalline } \mathrm{H}_{2} \mathrm{O}, f_{\mathrm{w}}=300 \% \\
80 \lesssim T_{\mathrm{d}} \lesssim 140 \mathrm{~K}^{8}\end{array}$}} \\
\hline Ice mantle & & \\
\hline Region & & \\
\hline \multicolumn{3}{|c|}{ Cold grains $^{6}$} \\
\hline Size & \multirow{3}{*}{\multicolumn{2}{|c|}{$\begin{array}{l}0.005 \leq a \leq 0.45 \mu \mathrm{m}, n(a) \propto a^{-3.5} \\
\text { amorphous } \mathrm{H}_{2} \mathrm{O}, f_{\mathrm{c}}=5 \% \\
T_{\mathrm{d}} \lesssim 80 \mathrm{~K}^{9}\end{array}$}} \\
\hline Ice mantle & & \\
\hline Region & & \\
\hline \multicolumn{3}{|c|}{ Disk $^{10}$} \\
\hline$R_{\text {in }}$ & $30 \mathrm{AU}$ & Adopted $^{11}$ \\
\hline$R_{\text {disk }}$ & $2000 \mathrm{AU}$ & $2000-3000$ \\
\hline \multirow[t]{2}{*}{$M_{\text {disk }}$} & $0.007 M_{\odot}\left(\mathrm{SH}^{12}, \mathrm{TO}^{13}\right)$ & $0.007-0.01$ \\
\hline & $0.05 M_{\odot}\left(\mathrm{LA}^{14}\right)$ & $0.03-0.07$ \\
\hline$H$ & $2.0(\mathrm{SH}), 1.0(\mathrm{TO}, \mathrm{LA})$ & $1.0-2.0$ \\
\hline$\dot{M}_{\mathrm{acc}}$ & $2.1 \times 10^{-7} M_{\odot} \mathrm{yr}^{-1}$ & 15 \\
\hline$\alpha, \beta$ & $15 / 8,9 / 8(\mathrm{SH}), 2,1$ (TO), 1,1 (LA) & Adopted \\
\hline \multicolumn{3}{|c|}{ Infalling envelope $\mathrm{e}^{16}$} \\
\hline$R_{\text {out }}$ & $0.5 \mathrm{pc}$ & $0.3-0.5$ \\
\hline$\dot{M}_{\text {env }}$ & $1.0 \times 10^{-4} M_{\odot} \mathrm{yr}^{-1}$ & $1.0-1.5 \times 10^{-4}$ \\
\hline$\mu_{0}$ & $\cos \left(65^{\circ} / 2\right)$ & Estimated \\
\hline$M_{\text {env }}$ & $177 M_{\odot}$ & $68.6-177^{17}$ \\
\hline \multicolumn{3}{|c|}{ Optical depth ${ }^{18}$} \\
\hline$\tau_{V, \mathrm{eq}}$ & $190(\mathrm{SH}), 210(\mathrm{TO}), 210(\mathrm{LA})$ & 19 \\
\hline$\tau_{V}$ & $66(\mathrm{SH}), 77$ (TO), 78 (LA) & 20 \\
\hline$\tau_{H}$ & $17(\mathrm{SH}), 20(\mathrm{TO}), 21(\mathrm{LA})$ & 20 \\
\hline$\tau_{K}$ & $10(\mathrm{SH}), 12(\mathrm{TO}), 12(\mathrm{LA})$ & 20 \\
\hline
\end{tabular}

${ }^{1}$ Ranges give the uncertainty of the corresponding model parameters; ${ }^{2}$ see Sect. 4.1.1; ${ }^{3}$ Kastner et al. (1992); ${ }^{4}$ Bernasconi \& Maeder (1996); Yorke \& Sonnhalter (2002); ${ }^{5}$ Kastner et al. (1992); ${ }^{6}$ see Sect. 4.2.1, the upper limit of the grain size of $0.45 \mu \mathrm{m}, f_{\mathrm{w}}$, and $f_{\mathrm{c}}$ are estimated; $730 \lesssim R \lesssim 1500 \mathrm{AU}(\mathrm{SH}, \mathrm{TO})$, or $1800 \mathrm{AU}(\mathrm{LA}) ;{ }^{8} 1500 \lesssim R \lesssim$ $5000 \mathrm{AU}(\mathrm{SH}, \mathrm{TO}), 1800 \lesssim R \lesssim 5000 \mathrm{AU}(\mathrm{LA}) ;{ }^{9} R \gtrsim 5000 \mathrm{AU}, 10$ : see Sect. 4.2.2; ${ }^{11}$ Monnier \& Millan-Gabet (2002); ${ }^{12}$ Shakura \& Sunyaev (1973); Pringle (1981); ${ }^{13}$ Toomre (1982); Lazareff et al. (1990); Fischer et al. (1996); ${ }^{14}$ Larson et al. (1984); ${ }^{15}$ derived from Eq. (5) in Whitney et al. (2003) with a disk viscosity of $0.01 ;{ }^{16}$ Ulrich (1976); Cassen \& Moosman (1981); Terebey et al. (1984); ${ }^{17}$ values correspond to $R_{\text {out }}$ of 0.3 and $0.5 \mathrm{pc}$, respectively, and $\dot{M}_{\text {env }}$ of $1.0 \times 10^{-4} M_{\odot} \mathrm{yr}^{-1} ;{ }^{18}$ calculated values obtained from our Monte Carlo code; ${ }^{19}$ the optical depths at $V$ band towards the equatorial plane; ${ }^{20}$ the optical depths at $V, H$, and $K$ bands towards the observer at a viewing angle of $\theta_{\mathrm{v}}=70^{\circ}$.

adopted values, and the ranges of uncertainty are summarized in Table 1.

\subsubsection{Dust temperature and SED}

Figure 6 displays the mass density distribution maps (top panels) and the dust temperature distribution maps (bottom panels) of the $\mathrm{SH}, \mathrm{TO}$, and LA disk models, which are presented in 

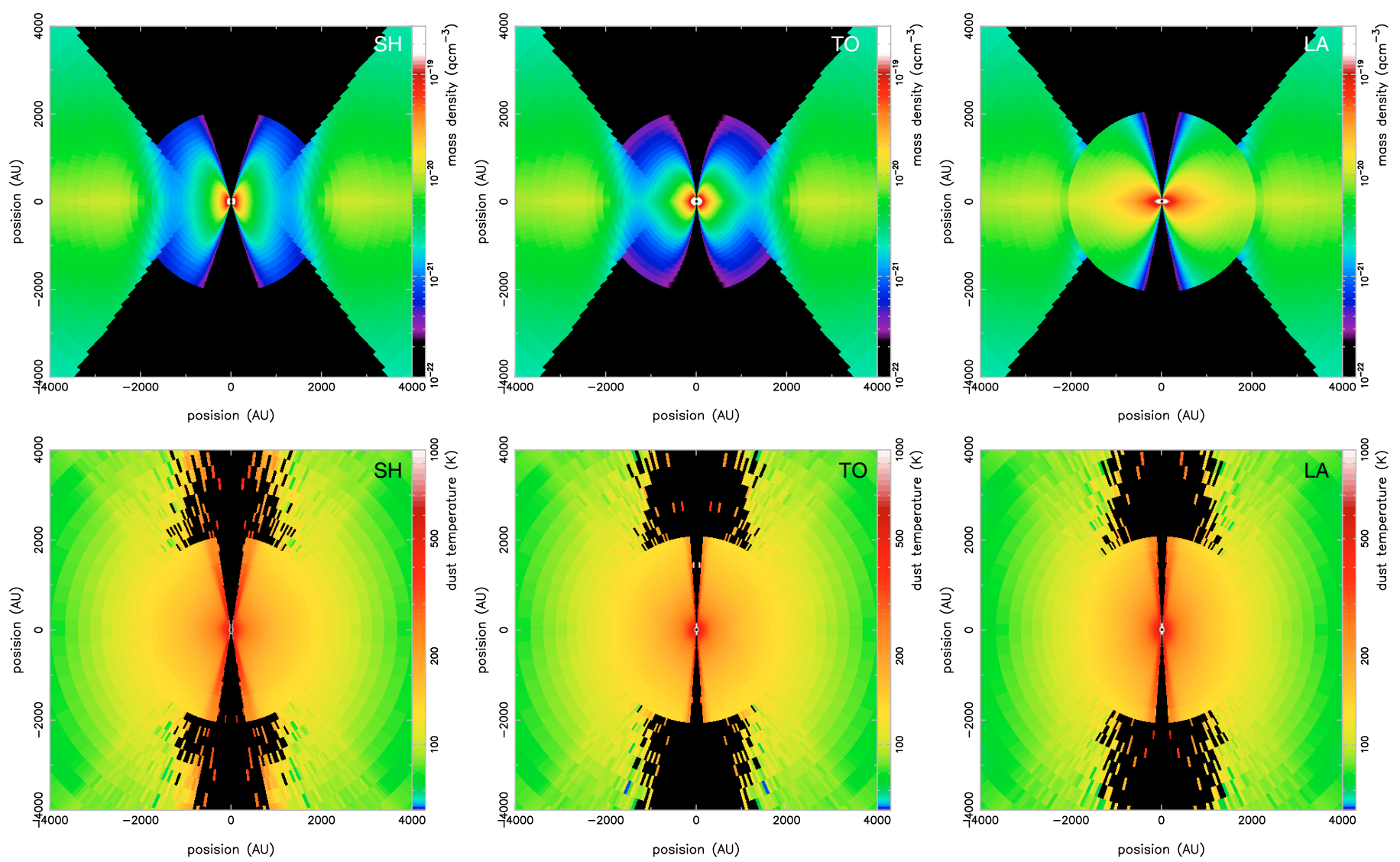

Fig. 6. Mass density distribution (top panels) and dust temperature distribution (bottom panels). The results of three potential disk models of the SH, TO, and LA are shown in the left, middle, and right columns, respectively. The dust temperature distribution maps are generated with the standard full interacting mode and $3 \times 10^{8}$ photon packets. Although the dust temperature in several cells in the polar direction still suffers from Monte Carlo noise, this does not affect the resulting SED significantly since the noise appears mainly in the low density region (cf. the corresponding mass density distribution maps).

the left, middle, and right columns, respectively. The dust density distribution of the disk determines the temperature structure. CRL 2136's disk has a very puffed-up or even a spherical shelllike structure with a thin polar hole $(H=1)$ and is extended $\left(R_{\text {disk }}=2000 \mathrm{AU}\right)$. Thus, the disk is heated nearly isotropically and efficiently even in the midplane. Although the SH model has a flared structure, the effect is seen only slightly in the polar region. An $\mathrm{H}_{2} \mathrm{O}$ maser observation of this object obtained evidence for a warm dust environment in the inner part of the disk (Menten \& van der Tak 2004). In their maser maps, most maser spots are seen within $200 \mathrm{AU}\left(=0\right.$.' $\left.^{\prime}\right)$, where the estimated dust temperature reaches $\gtrsim 350 \mathrm{~K}$ in our model. This is in good agreement with a prediction of $\sim 400 \mathrm{~K}$ by an excitation calculation (Elitzur et al. 1989). Furthermore, the above dust temperature and density distributions also affect the formation and growth of icy molecules. While the estimated dust temperature in the disk is $\gtrsim 100 \mathrm{~K}$ and the optical depth of grains with a water ice mantle at $3.08 \mu \mathrm{m}$ in the disk is found to be only 0.14 , the residual large fraction is calculated to be $\sim 11$ and occurs in the envelope. Thus, only a little water ice exists in the disk, which is consistent with the argument by Holbrook \& Temi (1998). The abovementioned disk properties of CRL 2136 are contrast with those of the T Tauri and Herbig Ae stars (e.g. Kenyon et al. 1993a; Calvet et al. 1994; Chiang et al. 2001).

Figure 7 presents comparisons of SEDs of the three potential (top panel) and two rejected (bottom panel) disk models with observations. The interstellar extinction is taken into account because CRL 2136 is located towards the Galactic plane $\left(b=+0.15^{\circ}\right)$. We estimate the visual extinction $A_{\mathrm{V}}$ to be 3.2 mag from a model of the general Galactic interstellar extinction (Allen 1973) for the distance of $2 \mathrm{kpc}$. The observed SED was corrected for this value by applying an extinction correction using the cross sections of the MRN grain model for the interstellar diffuse cloud (Mathis et al. 1977). The effects of aperture size should be considered. The aperture size of the $J H K$ band photometry obtained by Allen et al. (1977) is not specified. However, the $2.2 \mu \mathrm{m}$ flux is within the error compared to that obtained using a 24" aperture (Kastner et al. 1992), which probably captures the greatest fraction of flux from the source. Thus, we believe that Allen's photometric data suggests the real flux. Since the flux of Willner's spectrum at $2.2 \mu \mathrm{m}$ is in good agreement with Allen's $K$ band photometry, we also use this data without a flux correction. An infinite aperture size is applied in the computation of our model SED. It is thus also valid to directly compare our model SED to the above observations.

Because the dust temperature in the disk is higher than about $100 \mathrm{~K}$, the fluxes in the wavelengths shorter than $30 \mu \mathrm{m}$ are mainly determined by the dust density structure of the disk. We see the effect on the SEDs of the examined disk models. In the order of the TO, SH, LA, RB, and SL disk models, the dust concentrates towards the central star more, so that the mass of the higher temperature dust component is higher. Thus, the 4 to $10 \mu \mathrm{m}$ flux of the TO model was obtained to be higher than the flux of other models, as seen in Fig. 7 (top panel). In the RB and SL models, we find that a model with a $R_{\text {disk }}$ of $2000 \mathrm{AU}$, a $H$ of 1 , and a $M_{\text {disk }}$ of $0.07 M_{\odot}$ results in less discrepancy in the 

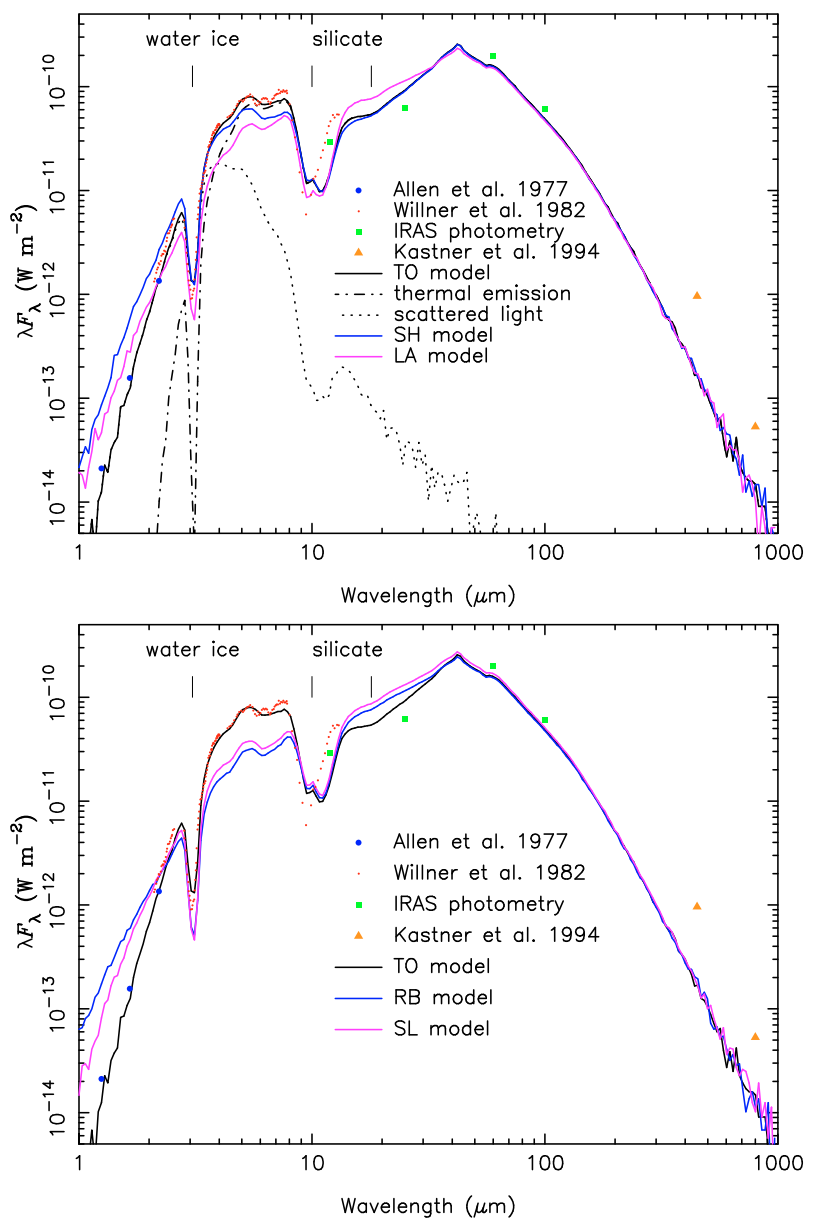

Fig. 7. Comparisons of SEDs of the acceptable, SH, TO, and LA disk models (top panel) and the rejected, RB and SL disk models (bottom panel) with observations. The results of these three disk models are indicated with violet, black, and blue curves, respectively. For the TO disk model, fluxes due to the thermal emission and dust scattering are also shown with a dotted-dashed line and dotted line, respectively. The attenuated stellar flux is not presented because it is less than $10^{-14} \mathrm{Wm}^{-2}$. The observed data is obtained from Allen et al. (1977) for the JHK band photometry, Willner et al. (1982) for the spectrum from $2.0 \mu \mathrm{m}$ to $15 \mu \mathrm{m}$, IRAS photometry at $12,25,60$, and $100 \mu \mathrm{m}$, and Kastner et al. (1994) for the photometry in sub-millimeter wavelength. An estimated interstellar extinction of 3.2 mag at $V$-band is dereddened in the observations.

examined 1680 models. However, a sufficient MIR flux is not obtained in these two models (see Fig. 7, bottom panel) for the above-mentioned reason. Thus, we reject these two disk models.

The $3 \mu \mathrm{m}$ and $10 \mu \mathrm{m}$ features would obtain better fits if the chemical compositions of water ice, methanol (see also Skinner et al. 1992), and silicate, and their fractions were more precisely chosen. The model flux around a wavelength of $60 \mu \mathrm{m}$ depends on the stellar luminosity but not on the above parameters much. Thus, our model result confirms that the estimated stellar luminosity of $5.5 \times 10^{4} L_{\odot}\left(1.4 \times 10^{4}[d / \mathrm{kpc}]^{2} L_{\odot}\right)$ is reasonable at a distance of $2 \mathrm{kpc}$.

\subsubsection{Intensity and polarization images}

Figure 8 presents the intensity images and the degree of polarization images in the $H$ and $K$ bands for the $\mathrm{SH}$, TO, and LA disk models. The polarization vectors are overlaid on the intensity images. In the intensity images, the central star feature and a V-shaped feature due to the cavity wall, which corresponds to the E and S1 lobes in our intensity images in Fig. 1, are reproduced. Although Fischer et al. (1996) reported that the appearance of the nebulosity near the central star differs depending on the disk form, our results show less difference, unlike the SED. This is probably due to the fact that Fischer's modeling made the comparisons using a constant mass density coefficient. In contrast, our modeling uses parameters which provide a good fit in the SED. A detailed analysis of the SED is also important in order to derive the disk geometry, as is being done to distinguish flat, flared, or self-shadowed structures in low mass and Herbig Ae stars (e.g. Meeus et al. 2001; Dullemond \& Dominik 2004). While the absolute fluxes in the V-shaped feature and towards the central star are in rough agreement with our observations, the model flux in the projected inner part of the cavity is higher than in the observations. This is obviously due to an asymmetric structure in the real CRL 2136 nebula. The lobe expected at the bottom is not visible in our model results because of the inclination effect.

In the polarization images, the degree of polarization is enhanced along the $\mathrm{V}$-shaped feature, as seen in our observations. The value is consistent with the observational results in the $H$ and $K$ bands, suggesting that our single scattering modeling provides a reasonable estimation of the grain sizes in the nebula. The centro-symmetric polarization vector pattern in the lobes and the polarization disk along the equatorial plane are reproduced in our models. However, the polarization vector alignment across the central star is not reproduced in our model. Three possible mechanisms have been proposed so far; (1) the PSF smoothing effect (Piirola et al. 1992); (2) multiple scattering at the boundaries of a geometrically thin disk (Bastien \& Ménard 1988); and (3) linear dichroism by aligned non-spherical grains (Hough et al. 1989). Because (1) our model images are convolved with a Gaussian function with the estimated PSF size in our observations; and (2) the immediate surrounding nebulosity of the central star looks somewhat spherical instead of a butterfly-like shape with a dark lane, the PSF smoothing effect can probably be ruled out. Furthermore, the possibility of multiple scattering at disk boundaries can also be ruled out in the case of CRL 2136 because the presence of a geometrically thin, optically thick disk is not expected based on our modeling. A third possibility remains. A higher polarization in the $H$ band $\left(P_{H}=32 \%\right)$ is present at IRS 1 compared to the $K$ band $\left(P_{K}=21 \%\right)$. This can be explained by dichroic absorption due to aligned, non-spherical grains in such a high extinction $\left(A_{\mathrm{V}} \sim 83\right)$ environment (e.g. Whittet et al. 2008) but not by scattering or the PSF smoothing effect. Therefore, for CRL 2136, we favor the explanations by Hough et al. (1989).

Interestingly, our high-resolution $J H K$-band imaging polarimetry of the Herbig Be star R Mon (Murakawa et al. 2008b) and the low mass protostar HL Tau (Murakawa et al. 2008c) show polarization behaviors very different from the above result of the massive star CRL 2136. In these objects, the polarization disk has significantly lower polarizations of $P_{J}=7 \%$ and $P_{H, K}<2 \%$ for R Mon and $P_{J, H, K}<3 \%$ for HL Tau. Such low polarizations are probably due to large grains (micron-sized or even larger) in the disks of these objects, while high polarizations in CRL 2136 are a result of small grains $\left(a_{\max }=0.45 \mu \mathrm{m}\right)$.

\section{Discussion and conclusion}

We presented $H$ - and $K$-band polarimetric images of the massive proto-stellar object CRL 2136 obtained using the Subaru telescope. The intensity images present two bright lobes extending 

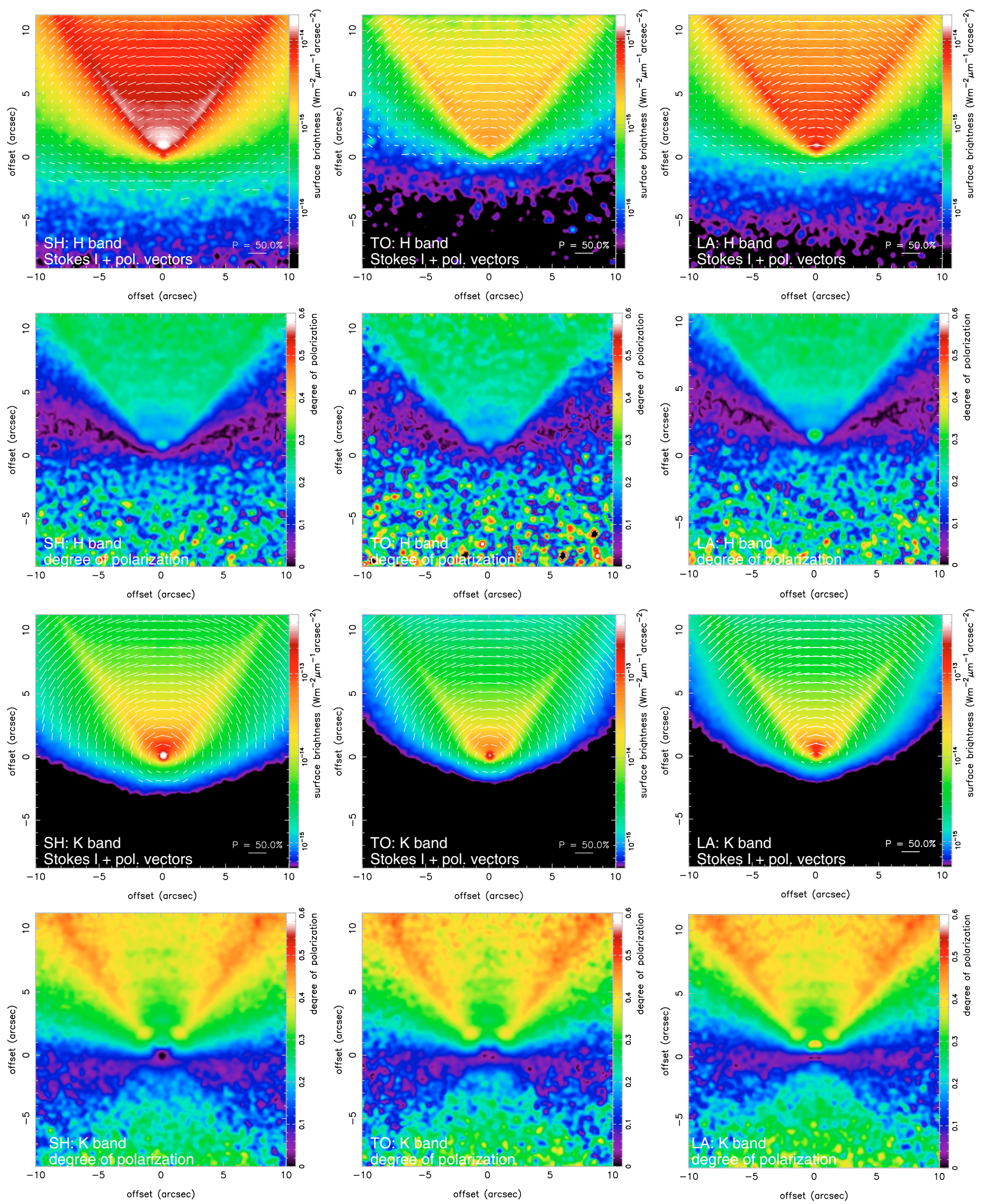

Fig. 8. Our model results for the intensity images, polarization vector lines, and the degree of polarization images. The top two rows represent the $H$ band and the bottom two rows the $K$-band. The left, middle, and right columns are results of the $\mathrm{SH}$, TO, and LA disk models, respectively. In these calculations, the scattering mode with the peeling-off technique and $1 \times 10^{8}$ photon packets were applied. All images were convolved with a Gaussian function with a full width at half maximum of 0 ' 35 , which corresponds to the PSF size of our observations. 
east and south and two faint lobes extending west and northwest, with respect to the central star IRS 1 . The polarization images show (1) centro-symmetrically aligned polarization vectors in all the lobes surrounding the central star; (2) high polarizations of $P_{H} \sim 30 \%$ and $P_{K} \sim 50 \%$ in the two bright lobes and low polarizations of $P_{H}<15 \%$ and $P_{K}<30 \%$ in the fan-shaped region between IRS 1 and the eastern and southern lobes; and (3) a polarization disk around the central star oriented along the PA of $\sim 45^{\circ}$; i.e. perpendicular to the direction of $\mathrm{CO}$ outflow. These results are consistent with previous observations (Minchin et al. 1991; Kastner et al. 1992) but are detected more clearly due to a higher angular resolution in our data. These signatures are convincing evidence that the central star of CRL 2136 is surrounded by an optically thick dust disk and an envelope with a polar cavity. Furthermore, our polarization data also show that the polarization vectors are aligned along the disk direction and gives a degree of polarization of $32 \%$ in the $H$ band and $21 \%$ in the $K$ band at IRS 1 .

In order to investigate the physical properties of the dust environment of CRL 2136, we performed radiative transfer modeling with 2-D disk and envelope geometries using our newly developed Monte Carlo code. Assuming three grain models with a DL-type chemistry with or without a water ice mantle, depending on the dust temperature, we found the maximum grain core size of $a_{\max }=0.45 \mu \mathrm{m}$ for all grain species, volume ratios of amorphous water ice of 0.05 for cold grains $\left(T_{\mathrm{d}} \lesssim 80 \mathrm{~K}\right)$, and crystalline water ice of 3.0 for warm grains $\left(80 \lesssim T_{\mathrm{d}} \lesssim 140 \mathrm{~K}\right.$ ). For the disk model, we found that the TO model, where the dust concentrates towards the central star the most, is the favored. Our modeling predicts that the disk has a low mass $\left(M_{\text {disk }}=0.007 M_{\odot}\right)$, puffed up $(H=1.0)$, and expanded $\left(R_{\text {disk }}=2000 \mathrm{AU}\right)$ structure. With our grain models assuming spherical shapes, the polarization vector alignment and high polarization in the polarization disk were not reproduced. The most plausible reason would be dichroic absorption of aligned, nonspherical grains, which probably exist in the equatorial, highdensity region in the inner part of the envelope. Further detailed studies involving treatment of spheroidal grains would provide important clues about the magnetic field structure in the disk as well as the envelope.

Our model results provide new information about the dust environment of CRL 2136. Previous observations of massive stars reported disk mass ranges of $\lesssim 0.1 M_{\odot}$ to $\sim 1 M_{\odot}$; e.g. Ori IRc2; Plambeck et al. (1995); Blake et al. (1996), Cep A; Patel et al. (2005), and M 17; Steinacker et al. (2006). These results might have implications for the evolutionary stage of the system, although some of these differences could be due to the use of different methods to estimate the disk parameters (e.g. a rotating component from a radio continuum, a flux distribution from mid-IR images, and radiative transfer modeling). A massive disk of $\sim 1 M_{\odot}(\sim 10 \%$ of the central object $)$ is likely due to gravitational instability in the early phase of star formation (e.g. Krumholz et al. 2005). In contrast, CRL 2136 has a low mass, a puffed-up disk, and a low disk accretion rate. This can be interpreted as follows: (1) the central star of $20 M_{\odot}$ formed rapidly $\left(\sim 2 \times 10^{5} \mathrm{yr}\right)$ with a high infalling rate and nearly isotropically $(H \sim 1)$ in the beginning of the star formation process; $(2)$ Then, after ignition of the central star, the strong radiation pressure from the luminous central star halted the accretion of the disk matter, resulting in a low mass, sparse disk structure keeping a nearly constant mass. Furthermore, these disk properties also affect the grain growth. In order for grains to grow in the disk, they have to undergo several grain-grain collisions in a high-density region. While it is believed that the disks around $\mathrm{T}$ Tauri stars and Herbig Ae stars provide such an environment, this does not seem to be the case for CRL 2136, according to our analysis. Therefore, grain growth is unlikely in CRL 2136's disk.

Acknowledgements. Our $H K$-band imaging polarimetry using the Subaru telescope was carried out on the Director's Discretionary Time. We would like to thank Prof. H. Karoji for providing us with the valuable opportunity. We also wish to thank the observatory staff for supporting our observations.

\section{References}

Allen, C. W. 1973, Astrophysical Quantities, 3rd edn. (London: Athlone Press), 263

Allen, D. A., Hyland, A. R., Longmore, A. J., et al. 1977, ApJ, 217, 108

Alvarez, C., Hoare, M., \& Lucas, P. 2004, A\&A, 419, 203

Bastien, P., \& Ménard, F. 1988, ApJ, 326, 334

Beckwith, S. V. W., \& Sargent, A. I. 1991, ApJ, 381, 250

Bernasconi, P. A., \& Maeder, A. 1996, A\&A, 307, 829

Bertie, J. E., Labbé, H. J., \& Whalley, E. 1969, J. Chem. Phys., 50, 4501

Bohren, C. F., \& Huffmann, D. R. 1983, Absorption and scattering of light by small particles (New York: John Wiley \& Sons)

Bjorkman, J. E., \& Wood, K. 2001, ApJ, 554, 615

Blake, G. A., Mundy, L. G., Garlstrom, J. E., et al. 1996, ApJ, 472, L49

Brooke, T. Y., Sellgren, K., \& Geballe, T. R. 1999, ApJ, 517, 883

Calvet, N., Hartmann, L., Kenyon, S. J., \& Whitney, B. A. 1994, ApJ, 434, 330

Cardelli, J. A., Clayton, G. C., \& Mathis, J. S. 1989, ApJ, 345, 245

Cashwell, E. D., \& Everett, C. J. 1959, A Practical Manual on the Monte Carlo Method for Random Walk Problems (New York: Pergamon Press)

Cassen, P., \& Moosman, A. 1981, Icarus, 48, 353

Chiang, E. I., Joung, M. K., Creech-Eakman, M. J., et al. 2001, ApJ, 547, 1077

Clayton, G. C., Wolff, M. J., Sofia, U. T., Gordon, K. D., \& Misselt, K. A. 2003, ApJ, 588, 871

Dartois, E., d'Hendecourt, L., Thi, W., Pontoppidan, K. M., \& van Dischoeck, E. F. 2002, A\&A, 394, 1057

d'Hendecourt, L. B., \& Allamandola, L. J. 1986, A\&AS, 64, 453

Dougados, C., Rouan, D., Lacombe, F., Tiphene, D., \& Forveille, T. 1990, A\&A, 227,437

Draine, B. T., \& Lee, H. M. 1984, ApJ, 285, 89

Duley, W. W., \& Williams, D. A. 1981, MNRAS, 196, 269

Dullemond, C. P., \& Dominik, C. 2004, A\&A, 417, 159

Elitzur, M., Hollenbach, D. J., \& McKee, C. F. 1989, ApJ, 346, 983

Fischer, O., Henning, Th., \& Yorke, H. W. 1996, A\&A, 308, 863

Gibb, E. L., Whittet, D. C. B., Boogert, A. C. A., \& Tielens, A. G. G. M. 2004, ApJS, 151, 35

Gordon, K. D., Misselt, K. A., Witt, A. N., \& Clayton, G. C. 2001, ApJ, 551, 269

Hagen, W., Tielens, A. G. G. M., \& Greenberg, J. M. 1981, Chem. Phys., 56, 367

Harvey, P. M., Butner, H. M., Colome, C., Francesco, J. D., \& Smith, B. 2000, ApJ, 534, 846

Herbst, E., \& Leung, C. 1985, MNRAS, 222, 689

Holbrook, J. C., \& Temi, P. 1998, ApJ, 496, 280

Holloway, R. P., Chrysostomou, A., Aitken, D. K., Hough, J. H., \& McCall, A. 2002, MNRAS, 336, 425

Hough, J. H., Whittet, D. C. B., Sato, S., et al. 1989, MNRAS, 241, 71

Ivezić, Ž., \& Elitzur, M. 1997, MNRAS, 287, 799

Jones, A. P., \& Williams, D. A. 1984, MNRAS, 209, 955

Kastner, J. H., \& Weintraub, D. A. 1996, ApJ, 466, L103

Kastner, J. H., Weintraub, D. A., \& Aspin, C. 1992, ApJ, 389, 357

Kastner, J. H., Weintraub, D. A., Snell, R. L., et al. 1994, ApJ, 425, 695

Kenyon, S. J., Calvet, N., \& Hartmann, L. 1993a, ApJ, 414, 676

Kenyon, S. J., Whitney, B. A., Gomez, M., \& Hartmann, L. 1993b, ApJ, 414, 773

Kim, S.-H., Martin, P. G., \& Hendry, P. D. 1994, ApJ, 422, 164

Kraus, S., Balega, Y., Elitzur, M., et al. 2006, A\&A, 455, 521

Krumholz, M. R., McKee, C. F., \& Klein, R. I. 2005, ApJ, 618, L33

Larson, R. B. 1984, MNRAS, 206, 197

Larson, K. A., \& Whittet, D. C. B. 2005, ApJ, 623, 897

Lazareff, B., Pudritz, R. E., \& Monin, J.-L. 1990, ApJ, 358, 170

Leger, A., Gauthier, S., Defourneau, D., \& Rouan, D. 1983, A\&A, 117, 164

Lucas, P. W., \& Roche, P. F. 1998, MNRAS, 299, 699

Lucy, L. B. 1999, A\&A, 344, 282

Mathis, J. S., Rumpl, W., \& Nordsieck, K. H. 1977, ApJ, 217, 425

Meeus, G., Waters, L. B. F. M., Bouwan, J., et al. 2001, A\&A, 365, 476

Menten, K. M., \& van der Tak, F. F. S. 2004, A\&A, 414, 289

Merrill, K. M., Russell, R. W., \& Soifer, B. T. 1976, ApJ, 207, 763 
Minchin, N. R., Hough, J. H., Burton, M. G., \& Yamashita, T. 1991, MNRAS, 251,522

Monnier, J. D., \& Millan-Gabet, R. 2002, ApJ, 579, 694

Murakawa, K., Suto, H., Tamura, M., et al. 2004, PASJ, 56, 509

Murakawa, K., Suto, H., Oya, S., et al. 2005, A\&A, 436, 601

Murakawa, K., Nakashima, J., Ohnaka, K., \& Deguchi, S. 2007, A\&A, 470, 957

Murakawa, K., Ohnaka, K., Driebe, T., et al. 2008a, A\&A, 489, 195

Murakawa, K., Preibisch, T., Kraus, S., \& Weigelt, G. 2008b, A\&A, 488, L75

Murakawa, K., Oya, S., Pyo, T.-S., \& Ishii, M. 2008c, A\&A, submitted

Niccolini, G., Woitke, P., \& Lopez, B. 2003, A\&A, 399, 703

Nussenzveig, H. M., \& Wiscombe, W. J. 1980, Phys. Rev. Lett., 45, 1490

Ohnaka, K., Driebe, T., Hofmann, K.-H., et al. 2006, A\&A, 445, 1015

Patel, N. A., Salvador, C., Sridharan, T. K., et al. 2005, Nature, 437, 109

Pendleton, Y. J., Tielens, A. G. G. M., \& Werner, M. W. 1990, ApJ, 349, 107

Pendleton, Y. J., Tielens, A. G. G. M., Tokunaga, A. T., \& Bernstein, M. P. 1999, ApJ, 513, 294

Plambeck, R. L., Wright, M. C. H., Mundy, L. G., \& Looney, L. W. 1995, ApJ, 455, L189

Piirola, V., Scaltriti, F., \& Coyne, G. V. 1992, Nature, 359, 339

Preibisch, T., Ossenkopf, V., Yorke, H. W., \& Henning, T. 1993, A\&A, 279, 577

Preibisch, T., Balega, Y. Y., Schertl, D., \& Weigelt, G. 2003, A\&A, 412, 735

Pringle, J. E. 1981, ARA\&A, 19,137

Shakura, N. I., \& Sunyaev, R. A. 1973, A\&A, 24, 337
Skinner, C. J., Tielens, A. G. G. M., Barlow, M. J., \& Justtanont, K. 1992, ApJ, 399, L79

Smith, R. G., Sellgren, K., \& Tokunaga, A. T. 1989, ApJ, 344, 413

Steinacker, J., Chini, R., Nielbock, M., et al. 2006, A\&A, 456, 1013

Terebey, S., Shu, F. H., \& Cassen, P. 1984, ApJ, 286, 529

Tielens, A. G. G. M., \& Hagen, W. 1982, A\&A, 114, 245

Toomre, A. 1982, ApJ, 259, 535

Ulrich, R. K. 1976, ApJ, 210, 377

van Dishoeck, E. F. 2004, ARA\&A, 42, 119

Weigelt, G., Beuther, H., Hofmann, K.-H., et al. 2006, A\&A, 447, 655

Whitney, B. A., \& Hartman, L. 1993, ApJ, 402, 605

Whitney, B. A., Kenyon, S. L., \& Gomez, M. 1997, ApJ, 485, 703

Whitney, B. A., Wood, K., Bjorkman, J. E., \& Wolf, M. J. 2003, ApJ, 591, 1049

Whittet, D. C. B., Pendleton, Y. J., Gibb, E. L., et al. 2001, ApJ, 550, 793

Whittet, D. C. B., Hough, J. H., Lazarian, A., \& Hoang, T. 2008, ApJ, 674, 304

Willner, S. P., Gillett, F. C., Herter, T. L., Jones, B., et al. 1982, ApJ, 253, 174

Wiscombe, W. J. 1980, Appl. Opt., 19, 1505

Witt, A. N. 1977, ApJS, 35, 1

Wolf, S., \& Voshchinnikov, N. V. 2004, CoPhC, 162, 113

Wollfire, M. G., \& Cassinelli, J. P. 1986, ApJ, 310, 207

Wood, K., \& Reynolds, R. J. 1999, ApJ, 525, 799

Yorke, H., \& Sonnhalter, C. 2002, ApJ, 569, 846

Yusef-Zadeh, F., Morris, M., \& White, R. L. 1984, ApJ, 278, 186 